\title{
EL PENSAMIENTO COMPUTACIONAL EN LA ELECTRÓNICA: LAIMPORTANCIADEL SOFTWARE DE SIMULACIÓN EN LA COMPRENSIÓN DEL PRINCIPIO DE FUNCIONAMIENTO DE LOS COMPONENTES ELECTRÓNICOS COMPUTATIONAL THINKING IN ELECTRONICS: THE IMPORTANCE OF SIMULATION SOFTWARE IN UNDERSTANDING THE PRINCIPLE OF OPERATION OF ELECTRONIC COMPONENTS
}

Javier Albiter Jaimes

Universidad Tecnológica del Sur del Estado de México. E-mail: xaver.albiter@live.com ORCID: https:// orcid.org/0000-0001-8269-6344

Rafael Valentín Mendoza Mendez

Centro Universitário UAEM Temascaltepec. México. E-mail: rvmendozam@uaemex.mx ORCID: https://orcid.org/0000-0003-4420-426X

Ernesto Joel Dorantes Coronado Centro Universitário UAEM Temascaltepec. México. E-mail: ernestodorantesc@hotmail.com ORCID: https://orcid.org/0000-0003-1037-3575

Recepción: 04/06/2019 Aceptación: 17/10/2019 Publicación: 30/12/2019

\section{Citación sugerida:}

Albiter Jaimes, J., Mendoza Mendez, R.V. y Dorantes Coronado, E.J. (2019). El pensamiento computacional en la electrónica: la importancia del software de simulación en la comprensión del principio de funcionamiento de los componentes electrónicos. 3C TIC. Cuadernos de desarrollo aplicados a las TIC, 8(4), 85-113. doi: http://doi.org/10.17993/3ctic.2019.84.85-113 


\section{RESUMEN}

La educación es parte integrante de las nuevas tecnologías y eso es tan así que un número cada vez mayor de universidades en todo el mundo está exigiendo la alfabetización electrónica como uno de los requisitos en sus exámenes de acceso y de graduación, por considerar que es un objetivo esencial preparar a los futuros profesionales para la era digital en los centros de trabajo.

Es así que en los últimos años ha tomado gran relevancia el concepto y aplicación del pensamiento computacional, ya que como parte de su metodología se encuentra la utilización de software de simulación como apoyo para el aprendizaje y solución de problemas, dichos paquetes informáticos permiten explorar, analizar, comprender, correlacionar y experimentar en el mundo virtual soluciones que posteriormente pueden aplicarse para resolver un problema real, como en el caso de la electrónica en el diseño y programación de circuitos, cuyos contenidos curriculares pueden ser complejos y extensos debido a la gran cantidad de desarrollos e innovaciones que han surgido en las últimas décadas.

\section{PALABRAS CLAVE}

Pensamiento computacional, Software de simulación, Electrónica, Circuitos, Método pedagógico, Tecnología y educación. 
Ed. 31 Vol. 8 N.o 4 Diciembre 2019 - Marzo 2020 DOI: http://doi.org/10.17993/3ctic.2019.84.85-113

\section{ABSTRACT}

Education is an integral part of the new technologies and that is so that an increasing number of universities worldwide are demanding electronic literacy as one of the requirements in their entrance and graduation exams, considering that it is an essential objective to prepare future professionals for the digital era in the workplace.

Thus, in recent years the concept and application of computational thinking has taken great relevance, since as part of its methodology is the use of simulation software as support for learning and problem solving, such computer packages that detect, analyze, understand, correlate and experience in the world virtual solutions that may have problems to solve a real problem, as in the case of electronics in circuit design and programming, curricular contents can be complex and extensive due to the great number of developments and innovations that have emerged in recent decades.

\section{KEYWORDS}

Computational thinking, Simulation software, Electronics, Circuits, Pedagogic method, Technology and education. 


\section{INTRODUCCIÓN}

La época actual está sumergida en desarrollos tecnológicos sumamente dinámicos, lo que hoy es la sensación tecnológica al cabo de unas semanas queda rezagado por otro desarrollo de recién creación, dichos desarrollos incluyen productos tanto de la parte de software como hardware, como ejemplo de ello tenemos los teléfonos inteligentes, los cuales han sufrido una modificación total en su concepto original, el cual era comunicar en tiempo real a diferentes personas desde ubicaciones diferentes y remotas utilizando como medio el espectro electromagnético (voz), al día de hoy, un teléfono inteligente se considera una minicomputadora que cuenta con todas la características propias de un computador, tiene un sistemas operativo (el más común Android) montado sobre un hardware denominado PCB (Printed Card Board) que contiene los mismos componentes de CPU (unidad de procesamiento central), dicho dispositivo inteligente te permite realizar llamadas, video llamadas en tiempo real, capturar esos momentos especiales por medio de la cámara fotográfica o la cámara de video, cuenta con diferente paquetería (App) la cuales permiten crear recordatorios, crear y manipular archivos digitales, entre otros.

Todas estas mejoras tecnológicas se han producido en un periodo corto de tiempo y así como los teléfonos han evolucionado, también lo han hecho a la par las computadoras (personales o de escritorio) junto con los sistemas operativos y sus distintas paqueterías. Todo este desarrollo tecnológico ha dado lugar para la creación de nuevos paquetes o programas informáticos los cuales han generado un cambio significativo en el actuar de nuestra vida diaria, hoy en día el uso de softwares es vital en los espacios laborales, por ejemplo, el uso de WhatsApp como medio de comunicación principal es algo que no se puede negar.

La Fundación Nacional para la Ciencia(NSF), por medio del Sociedad Internacional para la Tecnología en la Educación (ISTE) y la Asociación de profesores de informática(CSTA), impulsa activamente un nuevo enfoque de enseñanza para que en todos los niveles de educación se incluya el Pensamiento Computacional (Computational Thinking). Este nuevo enfoque busca promover en la educación el 
desarrollo de habilidades de pensamiento que conduzcan a la formación de personas orientadas a la creatividad y a la innovación (Zapotecatl López, 2018).

Con respecto al sistema educativo también se ha visto afectado o impactado como consecuencia de esta explosión tecnológica de la informática y este nuevo enfoque de enseñanza denominado Pensamiento computacional (PG en lo sucesivo), como consecuencia de lo anterior, dentro de la educación superior el uso de los diferentes paquetes informáticos es demasiado múltiple, dentro de este contexto se encuentra el software de simulación el cual se ha ido integrando en la última década como parte de las didácticas de la enseñanza educativa.

Dentro de la educación superior el uso de los diferentes paquetes informáticos es demasiado múltiple, dentro de este contexto se encuentra el software de simulación el cual se ha ido integrando en la última década como parte de las didácticas de la enseñanza educativa.

Pareja Aparicio (2013) menciona que:

La simulación nos sirve como punto intermedio entre los conceptos teóricos y la realidad. Cuanto mejor sea la expresión que defina a la realidad, mejores serán los resultados, porque serán más reales y, a su vez, nos puede reducir los costes de fabricación, facilitando las tareas de diseño (p.2).

Algunas de las ventajas que tiene la utilización de software de simulación según menciona Pareja Aparicio (2013) son:

- Reducción del tiempo de diseño, puesto que permite verificar el diseño sin tener que realizar el montaje en un sistema físico, así como facilitar que las modificaciones necesarias sean menores.

- Reducción de costos, como consecuencia de la reducción del tiempo de diseño, pero también porque permite comprobaciones sin tener que comprar los componentes previamente. 
- Modificación de los efectos internos y externos del sistema, y ver cómo afectan en su funcionamiento.

- Observación detallada del sistema que se está simulando, lo que puede conducir a un mejor entendimiento del sistema. Esto resulta muy útil en la formación para la comprensión de la elección de los valores de los componentes que intervienen en un circuito.

- Experimentación con nuevas situaciones, sobre las cuales se tiene poca o ninguna información. A través de esta experimentación se pueden anticipar mejor posibles resultados no previstos.

- Anticipación de posibles problemas que pueden surgir en el comportamiento del sistema cuando nuevos elementos son introducidos.

\section{ESTADO DEL ARTE}

Adell, Llopis, Esteve, y Valdeolivas (2018) señalan que en los últimos años, el PC ha irrumpido con fuerza en el debate sobre el currículo de la educación obligatoria de numerosos países de nuestro entorno. En el informe del Joint Research Center de la Unión Europea (Bocconi, Chioccariello, Dettori, Ferrari y Engelhardt, 2016) que ha analizado dichas políticas en la Unión Europea y otros países se afirma que dos grandes tendencias emergen como justificación de la necesidad de su integración. Por una parte, el desarrollo de habilidades de PC en niños y jóvenes debe facilitar que "puedan pensar de manera diferente, expresarse a través de una variedad de medios, resolver problemas del mundo real y analizar temas cotidianos desde una perspectiva diferente" (Bocconi et al., 2016, p. 25). Por otra, su integración es necesaria "para impulsar el crecimiento económico, cubrir puestos de trabajo TIC y prepararse para futuros empleos" (Bocconi et al., 2016, p. 25). 
Se han identificado múltiples prácticas educativas para desarrollar el PG, pero todas ellas devienen de las propuestas de Seymour Papert y su teoría del construccionismo, que prioriza el aprender haciendo. En esa línea las experiencias más difundidas a nivel mundial son las desarrolladas desde el Instituto Tecnológico de Massachusetts MIT, que van desde la promoción de la programación desde los primeros niveles educativos, hasta el uso de la Robótica Educativa para lograrlo. "No basta solamente familiarizarse con el uso y manejo instrumental de las nuevas tecnologías, sino también incorporarlas a procesos de creación, innovación y gestión del conocimiento a través del pensamiento computacional" (Balladares Burgos, Avilés Salvador, y Pérez Narváez, 2016, pág. 152).

Algunas de las iniciativas ya existentes del pensamiento computacional según Zapata-Ros (2018) son las siguientes:

NUEVA ZELANDA: CS Unplugged es una colección de actividades de aprendizaje gratuitas que enseñan Ciencias de la Computación a través de interesantes juegos y acertijos, que usan tarjetas, cuerdas, lápices de colores y muchos juegos como los de Ikea o Montesori-Amazon. Fue desarrollado para que los jóvenes estudiantes puedan interactuar con la informática, experimentar los tipos de preguntas y desafios que experimentan los científicos informáticos, pero sin tener que aprender primero la programación.

SINGAPUR: Para abordar la creciente necesidad de nuevos programas de tecnología educativa (en este caso de Pensamiento Computacional a través fundamentalmente de robótica) en las aulas de la primera infancia, se lanzó el programa PlayMaker de Singapur. Es un programa en línea destinado a los maestros, para introducir a los niños más pequeños a la tecnología. Según Steve Leonard, vicepresidente de la Autoridad de Desarrollo de Infocomm de Singapur (IDA), "a medida que Singapur se convierta en una nación inteligente, nuestros hijos necesitarán sentirse cómodos creando con tecnología”. 
Aprovechando el creciente movimiento STEM, el objetivo del programa PlayMaker no es solo promover el conocimiento técnico sino también brindar a los niños herramientas para divertirse, practicar la resolución de problemas y generar confianza y creatividad (Chambers, 2015; Digital News Asia, 2015).

MACEDONIA: Jovanov, Stankov, Mihova, Ristov y Gusev, (2016) presentan en EDUCON, 2016 IEEE, una descripción general de una aportación al currículo macedónico, introducida en 2015, titulada "Trabajar con computadoras y conceptos básicos de programación” o simplemente "Computación", para abreviar. Ofrecen una visión general del estado de la educación informática en Macedonia antes de esta propuesta, hacen un análisis y luego ofrecen una visión general de la nueva materia introductoria para alumnos de ocho años. En su comunicación dan una visión general del contenido que incluye siete unidades que se impartirán en dos clases por semana.

En su trabajo Jovanov et al. (2016) comunican que en la iniciativa organizan los contenidos en siete unidades que se impartirán en dos clases por semana:

- Primeros pasos para usar la computadora

- Gráficos por computadora

- Procesamiento de texto

- Vida en línea

- Concepto de algoritmos y programas

- Pensamiento computacional a través de un juego

- Creación de programas simples 
Cárdenas Peralta (2019) presenta algunos modos de integración del curriculum de pensamiento computacional y programación:

INGLATERRA. En 2014, reemplazó la materia Information and Communication Technologies -Tecnologías de la Información y la Comunicación-, por Computing -Computación-, cuya principal innovación fue la inclusión del pensamiento computacional y la programación en escuelas primarias y secundarias. Esta materia, cuya esencia son las ciencias de la computación, remarca la importancia de la creatividad y del dominio de los sistemas digitales como recursos para cambiar el mundo y se propone como base para el desarrollo de las habilidades relacionadas con la alfabetización digital.

Los objetivos del plan de estudios están orientados a:

- Comprender y aplicar los principios y conceptos fundamentales de las ciencias de la computación, incluyendo la abstracción, la lógica, los algoritmos y la representación de datos.

- Analizar problemas en términos computacionales y escribir programas para resolverlos.

- Evaluar y aplicar tecnología de la información, incluyendo aquellas nuevas o poco familiares, para resolver problemas.

- Ser usuarios responsables, competentes, seguros y creativos de tecnologías de las TIC.

SUEGIA. Integró la programación en la educación obligatoria a partir de 2018. Esta medida fue impulsada tras una serie de orientaciones para la construcción de políticas públicas, en el marco del avance de la sociedad digital. En 2012, el Comité de Digitalización de Suecia incluyó, dentro de sus recomendaciones para el futuro del trabajo, la necesidad de enseñar la programación, tras lo cual el gobierno sueco, en 2015, solicitó a la Agencia Nacional de Educación desarrollar una estrategia digital para el sistema educativo, que contemplara una actualización curricular para su inclusión en la educación obligatoria (Heintz et al., 2017). Consecuentemente, la Agencia Nacional de Educación 
desarrolló, en 2017, un plan organizado en base a tres áreas principales: la competencia digital para todo el sistema educativo, la equidad en el acceso y uso, y la investigación y evaluación de las posibilidades de la digitalización

La implementación de la estrategia digital incluyó la revisión del currículum para la educación obligatoria, publicado en 2011, que entró en vigor a partir de septiembre de 2018. La programación fue incorporada de modo transversal, asociada a diferentes áreas de conocimiento; principalmente en la asignatura Matemática y, en menor medida, en las materias Tecnología y Educación cívica.

AUSTRALIA. El Consejo de Educación de Australia aprobó un nuevo plan de estudios en 2015, que presenta ocho áreas de aprendizaje, entre las cuales se incluye Tecnologías. Esta área, a su vez, reúne dos asignaturas distintas pero relacionadas entre sí: Tecnologías digitales, y Diseño y tecnologías. Estas materias buscan promover oportunidades para que los estudiantes comprendan cómo se crean las soluciones y cómo se usarán en el futuro, identificando sus posibles beneficios y riesgos.

Según establece el currículum australiano, el área Tecnologías tiene como objetivo desarrollar el conocimiento, la comprensión y las habilidades para garantizar que, individualmente y en colaboración, los estudiantes sean capaces de:

- Investigar, diseñar, planificar, gestionar, crear y evaluar soluciones.

- Ser creativos, innovadores y emprendedores, al usar tecnologías tradicionales, contemporáneas y emergentes, y entender cómo se han desarrollado las tecnologías a lo largo del tiempo.

- Informar y tomar decisiones éticas sobre el papel, el impacto y el uso de las tecnologías en la economía, el medio ambiente y la sociedad para un futuro sostenible.

- Participar con confianza y seleccionar y manipular responsablemente las tecnologías (materiales, datos, sistemas, componentes, herramientas y equipos), al diseñar y crear soluciones. 
- Criticar, analizar y evaluar problemas, necesidades u oportunidades para identificar y crear soluciones.

\section{METODOLOGÍA}

Para la realización del presente trabajo se utilizará una metodología documental, recordemos que el fin de la revisión de literatura consiste en detectar y obtener información y consultar bibliografia y otros materiales que pueden ser útiles para alcanzar el objetivo del estudio. Este registro es selectivo, puesto que cada año se publican en diversas partes del mundo cientos de miles de artículos en revistas y libros, además de materiales electrónicos (Jurado Rosas, 2005).

Cortes Rojas y Garcia Santiago (2012) mencionan que:

La revisión de la literatura consiste en detectar, obtener y consultar la bibliografía y otros materiales de utilidad para los propósitos de la investigación; es decir, para extraer y recopilar información relevante y necesaria para la investigación. Ésta debe ser selectiva, o sea, tratar de evitar el "hábito coleccionista" así como la "búsqueda a ciegas". Como no existe una guía que indique qué documentos son importantes y cuáles no lo son, lo fundamental es tener presente la finalidad de la investigación para evitar el fenómeno "bola de nieve", que en palabras de Ander Egg quiere decir que "un documento remite a otro y así sucesivamente, con lo cual se pueden encontrar pistas interesantes o quedar 'ahogado' y 'aplastado' por el afán de recopilar todo". (p.7)

\section{DESARROLLO}

En la actualidad las instituciones se han visto sorprendidos por un hecho: la sociedad y los sistemas de producción, de servicios y de consumo demandan profesionales cualificados en las industrias de la información. Particularmente en el mundo desarrollado se da la paradoja de países y regiones con un 
alto índice de paro en las que actualmente se quedan sin cubrir puestos de trabajo de ingenieros de software, desarrolladores de aplicaciones, documentalistas digitales, por falta de egresados de las escuelas técnicas, por falta de demanda de estos estudios por parte de potenciales alumnos y sobre todo por la falta de personal capacitado.

Zapata-Ros (2015) menciona que ante esta situación los sistemas educativos de los países más sensibles han abordado el problema desde la perspectiva de una reorganización del curriculum en la mayor parte de los casos donde se ha producido esa reacción. Sin embargo, la cuestión de fondo supone la aparición de unas nuevas destrezas básicas. Las sociedades más conscientes han visto que se trata de una nueva alfabetización, una nueva alfabetización digital, y que por tanto hay que comenzar desde las primeras etapas del desarrollo individual, al igual como sucede con otras habilidades clave: la lectura, la escritura y las habilidades matemáticas, e incluso estudiando las relaciones y coincidencias de esta nueva alfabetización con estas competencias claves tradicionales.

Las sociedades más conscientes han visto que se trata de una nueva alfabetización, una nueva alfabetización digital, y que por tanto hay que comenzar desde las primeras etapas del desarrollo individual.

La educación es parte integrante de las nuevas tecnologías y eso es tan así que un número cada vez mayor de universidades en todo el mundo está exigiendo la alfabetización electrónica como uno de los requisitos en sus exámenes de acceso y de graduación, por considerar que es un objetivo esencial preparar a los futuros profesionales para la era digital en los centros de trabajo. 


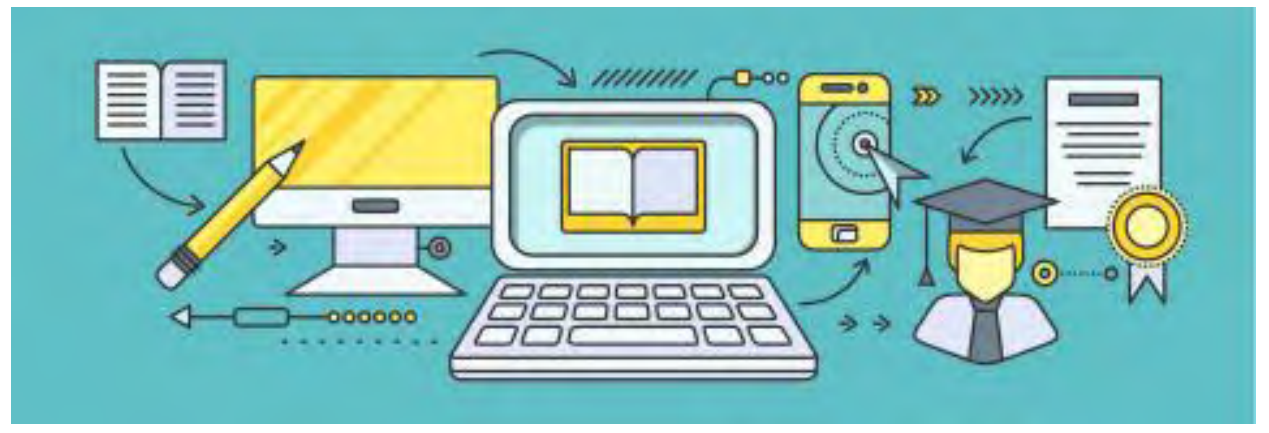

Figura 1. Elearning. Fuente: (Datos.gob.es, 2018).

La mayoría de las instituciones de educación superior cuentan, en mayor o menor medida, con equipos informáticos que posibilitan el acceso a Internet de los alumnos. Así, los universitarios, incluso aquellos que por problemas económicos no cuentan con computadores en sus hogares, pueden acceder a un mundo que antes era exclusivo de las clases pudientes, teniendo la oportunidad de visitar museos y accediendo a conocimientos disponibles gratuitamente. Es en este sentido, que el papel del profesor universitario es fundamental: Cuanto más se inculque en los universitarios la posibilidad de utilizar las nuevas tecnologías, más amplio será el mundo que obra para ellos y las oportunidades que tengan de encontrar trabajo (Rosario, 2006).

Toda esta explosión logarítmica de nueva tecnología ha generado un cambio en los paradigmas de la enseñanza, lo que ha generado optar por nuevas metodologías de aprendizaje en pro de la mejora del proceso de enseñanza-aprendizaje, el Pensamiento Computacional es una nueva metodología de enseñanza la cual tiene como objetivo principal la resolución de problemas desde un enfoque de la programación computacional que se basa en el análisis y diseño de algoritmos.

La investigadora Jeannette Wing lo define como: "los procesos de pensamiento involucrados en la formulación de problemas y representación de sus soluciones, de manera que dichas soluciones puedan 
ser ejecutadas efectivamente por un agente de procesamiento de información (humano, computadora o combinaciones de humanos y computadoras)" (Zapotecatl López, 2018).

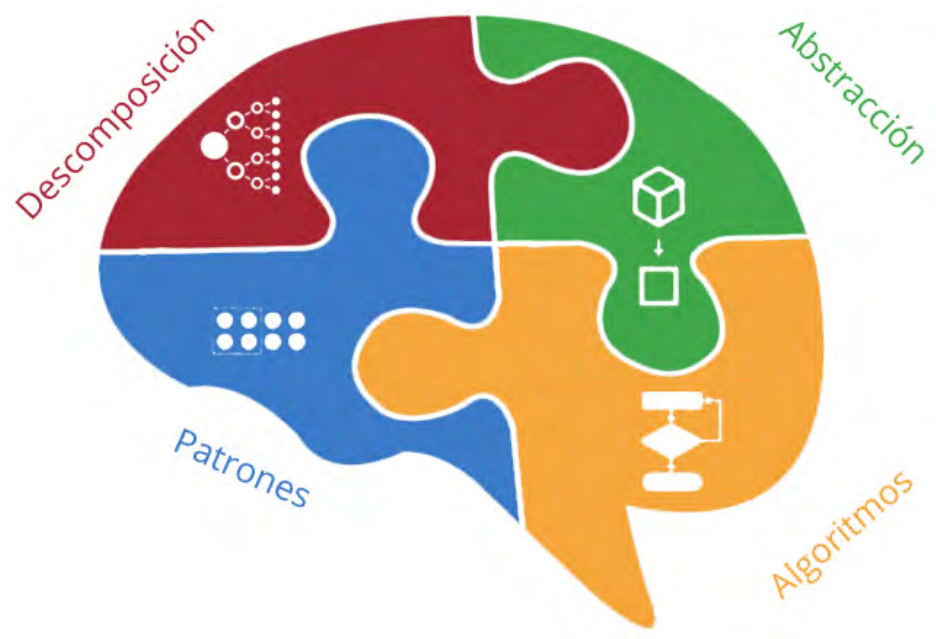

Figura 2. Los procesos del pensamiento computacional.

Otras definiciones han ido surgiendo en la literatura científica desde entonces. Entre las más aceptadas se encuentran la de Aho y la de la Royal Society (Moreno León, 2014):

- $\quad$ El PG es el proceso que permite formular problemas de forma que sus soluciones pueden ser representadas como secuencias de instrucciones y algoritmos.

- El PG es el proceso de reconocimiento de aspectos de la informática en el mundo que nos rodea, y aplicar herramientas y técnicas de la informática para comprender y razonar sobre los sistemas y procesos tanto naturales como artificiales.

El objetivo es desarrollar sistemáticamente las habilidades del pensamiento de orden superior, como el razonamiento abstracto, el pensamiento crítico y la resolución de problemas, con base en los conceptos 
de la computación. Además, potenciar el aprovechamiento del poder de cálculo que tienen las computadoras actualmente para innovar y volverlo una herramienta científica (Zapotecatl López, 2018).

Además, proporciona una estructura imprescindible para el estudio de la programación, que va más allá de la codificación en sí misma. Permite al estudiante enfrentar problemas, descomponerlos en elementos y encontrar algoritmos que los resuelvan. En consecuencia, el pensamiento computacional implica: descomposición, reconocimiento de patrones, abstracción, generalización de patrones y diseño algorítmico (Valverde Berrocoso, Fernández Sánchez, y Garrido Arroyo, 2015).

Figura 3. Generalización de patrones y diseño algorítmico.

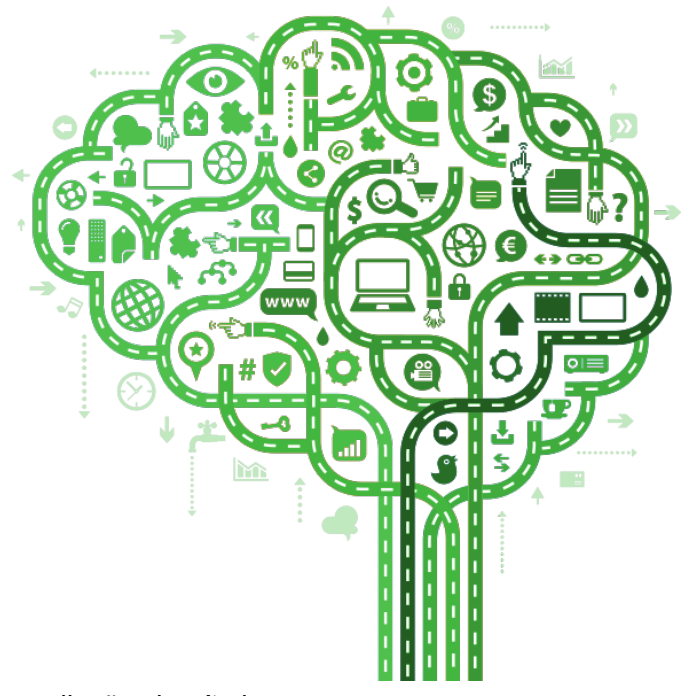

Como menciona CSTA \& ISTE (2011), el PG es un proceso de solución de problemas que incluye (pero no se limita a) las siguientes características:

- Formular problemas de manera que permitan usar computadores y otras herramientas para solucionarlos.

- Organizar datos de manera lógica y analizarlos. 
- Representar datos mediante abstracciones, como modelos y simulaciones.

- Automatizar soluciones mediante pensamiento algorítmico (una serie de pasos ordenados).

- Identificar, analizar e implementar posibles soluciones con el objeto de encontrar la combinación de pasos y recursos más eficiente y efectiva.

- Generalizar y transferir ese proceso de solución de problemas a una gran diversidad de estos.

Igualmente complementa y combina el pensamiento matemático y la ingeniería, porque se basa en las matemáticas como sus fundamentos y recurre a la ingeniería por la interacción de los sistemas con el mundo real. Estos sistemas están limitados por la física del dispositivo subyacente, pero por medio de la computadora se pueden construir simulaciones o mundos virtuales sin las restricciones de la realidad fisica.

Programar en la educación escolar constituye una buena alternativa para ayudar a los estudiantes a desarrollar habilidades de pensamiento de orden superior, especialmente pensamiento computacional. Desde el punto de vista educativo, el desarrollo de software posibilita no solo activar una amplia variedad de estilos de aprendizaje sino desarrollar el pensamiento computacional. Adicionalmente, compromete a los estudiantes en la consideración de varios aspectos importantes para la solución de problemas: decidir sobre la naturaleza del problema, descomponerlo en subproblemas más sencillos, seleccionar una representación algorítmica que ayude a resolver cada subproblema y, monitorear sus propios pensamientos (metacognición) y estrategias de solución (Vilanova, 2018).

$\mathrm{Al}$ escribir código los alumnos aprenden cómo organizar un proceso, reconocen rutinas o repeticiones y descubren errores en su pensamiento computacional cuando su programa no funciona según la idea o expectativa con la que fue concebido. Todas ellas son características clave del pensamiento 
computacional. Mediante la codificación se pueden construir aprendizajes significativos desde un punto de vista individual, social, cultural y tangible que conduzcan a una "participación computacional".

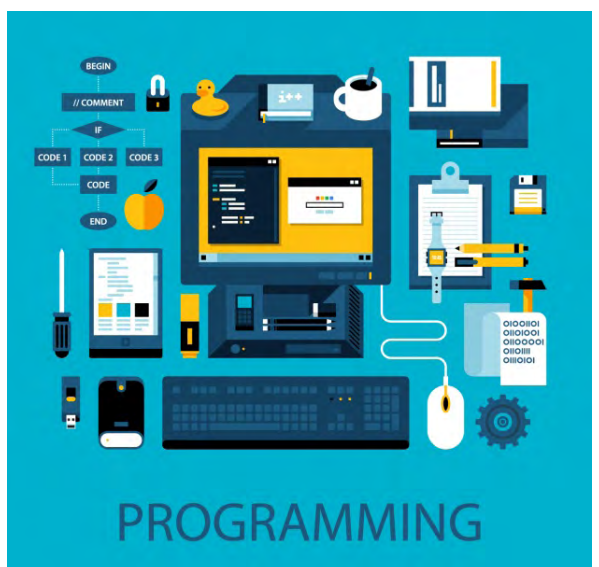

Figura 4. Desarrollo del pensamiento computacional a través de la programación.

Las TICs son un conjunto de procesos y productos derivados de las nuevas herramientas (Hardware y software), soportes de la información y canales de comunicación, relacionada con el almacenamiento, procesamiento y transmisión digitalizados de la información de forma rápida y en grandes cantidades.

“Con la incorporación de las TICs, el proceso de aprendizaje universitario deja de ser una mera recepción y memorización de datos recibidos en la clase, pasando a requerir una permanente búsqueda, análisis y reelaboración de informaciones obtenidas en la red. De este modo, el estudiante deja de ser sólo un procesador activo de información, convirtiéndose en un constructor significativo de la misma, en función de su experiencia y conocimientos previos, de las actitudes y creencias que tenga, de su implicación directa en el aprendizaje, y de que persiga el desarrollo de procesos y capacidades mentales de niveles superiores (Mayer, 2000)" (Ferro Soto y Martínez Senra, 2009).

Como parte de la metodología de PG se encuentra la utilización de software de simulación, dichos paquetes informáticos han permitido explorar, analizar, comprender, correlacionar y experimentar 
en el mundo de la electrónica, hoy en día hablar de electrónica es un tema demasiado complejo y extenso debido a la gran cantidad de desarrollos e innovaciones que han surgido en las últimas décadas, aumentado con ello la magnitud de conocimientos y literatura de la electrónica.

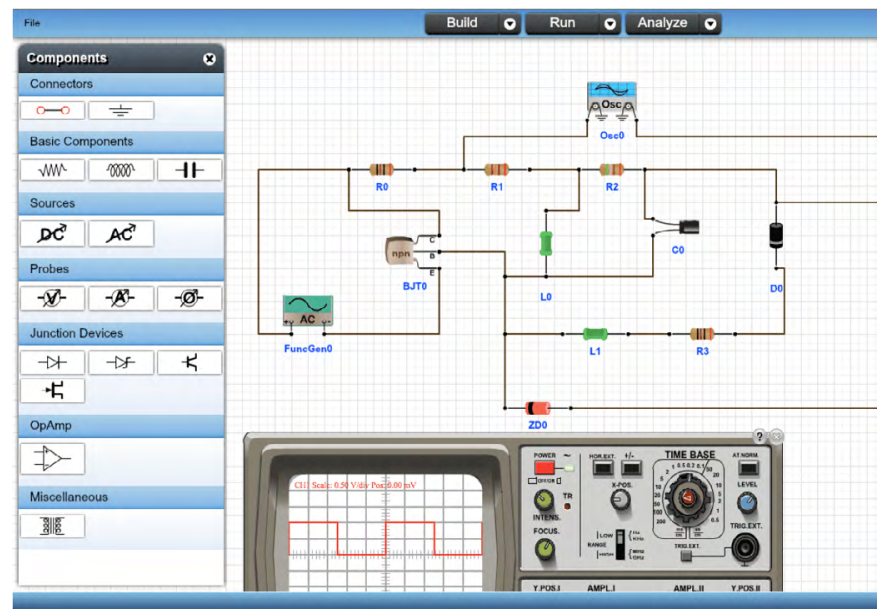

Figura 5. Software de electrónica DoCircuits. Full aprendizaje. (2019).

López (2016) menciona que existen diferentes tipos de actividades que pueden ser implementadas con el uso de herramientas computacionales, haciendo una escueta clasificación, de acuerdo con su uso, en actividades de animación, simulación y modelación. Desde su perspectiva, la animación permite la observación de una secuencia predeterminada, posibilitando (por decirlo de alguna manera) el sentir de los pensamientos de otros. Las simulaciones permiten al usuario hacer mediciones en la entrada de valores y observar como varían los resultados, consiguiendo con esto una comprensión funcional de los pensamientos de otros. Por último, este autor concibe la modelación como una actividad que permite el cambio de reglas y de entrada de valores, observar la variación en los resultados obtenidos, facilitar una lectura de los pensamientos de otros, dándole la posibilidad de hacer modificaciones para expresar su propio pensamiento. Desde la perspectiva de este autor, es la habilidad para alterar las reglas lo que 
se entiende como modelación, siendo esta condición la que la hace diferente de actividades como la animación o la simulación.

La simulación computacional es la conjunción de algoritmos matemáticos que modelan el comportamiento dinámico de sistemas físicos y herramientas computacionales que permiten reproducir y visualizar esta dinámica (Zapotecatl López, 2018).

La simulación computacional involucra implementar modelos a fin de realizar experimentos y probar hipótesis. Además, debido a que el programador tiene la libertad de ordenar en la simulación cualquier cosa que imagine, la simulación computacional también puede utilizarse para fines artísticos. Por ejemplo: el modelo de boids ha tenido aplicaciones en videojuegos y en las películas para proporcionar representaciones realistas de grupos moviéndose coordinadamente. En particular, la película Batman Returns utilizó el modelo de boids para simular bandadas de murciélagos y los ejércitos de pingüinos que caminan en las calles de Ciudad Gótica (Zapotecatl López, 2018).

Estas nuevas tecnologías hacen posible la simulación de secuencias y fenómenos físicos, químicos o sociales o fenómenos en 3D, entre otros, de manera que los estudiantes pueden experimentar con ellos y así comprenderlos mejor. Además, ponen a su disposición múltiples materiales para la autoevaluación de sus conocimientos. En definitiva, facilitan el aprendizaje a partir de los errores, permitiendo conocerlos justo en el momento en que se producen (feed back inmediato a las respuestas y a las acciones de los usuarios) y, generalmente, el programa ofrece la oportunidad de ensayar nuevas respuestas o formas de actuar para superarlos.

El modelo de boids ha tenido aplicaciones en videojuegos y en las películas para proporcionar representaciones realistas de grupos moviéndose coordinadamente, en particular, la película Batman Returns. 


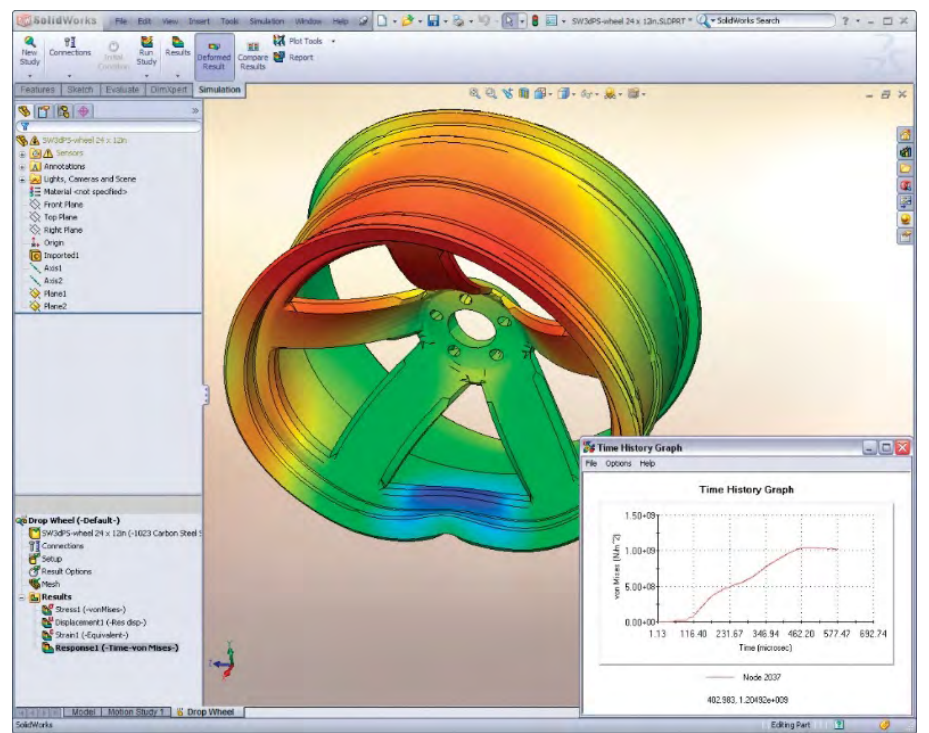

Figura 6. Simulación de una deformación en un rin de automóvil. Cuevana3 (2019). Fuente: (Cuevana, 2019) Recuperado de http://cuevana3.net/descargar-tutoriales-solidworks-gratis-curso-avanzado-descargar-gratis/

Uno de los elementos TIC que se usa con más frecuencia en las aulas son los simuladores. Se tratan de programas que trabajan con la computación numérica para reproducir de manera virtual escenarios estrechamente relacionados con la realidad. Durante el proceso de simulación el usuario puede manejar distintos parámetros de entrada, generando unos resultados de medida mensurables. Generalmente este tipo de herramienta representa gráficamente estos resultados de modo que el alumno puede relacionarlo directamente con la realidad y facilitar su aprendizaje.

La simulación y el aprendizaje son dos conceptos muy unidos en el proceso educativo. Desde el punto de vista puramente instrumental podemos decir que la mayoría de las actividades de aprendizaje siempre están basadas en entidades de simulación. Como recurso de aprendizaje, "la simulación puede generar un número de diferentes escenarios en respuesta a los cambios de parámetros que el usuario usa para categorizar la simulación, y poder producir una animación para ilustrar los resultados de este modelo. 
Una simulación puede usarse para extender un estudio de caso, y podría incluir clips de audio y vídeo y juegos de rol, así como gráficos basados en web y la construcción de escenarios” (Mason y Rennie, 2006, 106).

Como herramienta de apoyo al estudio presenta numerosas ventajas: favorece el aprendizaje por descubrimiento, obliga a demostrar lo aprendido, ejercitación del alumno de forma independiente, reproducir la experiencia un elevado número de veces con el mismo control de variables, permite al alumno reaccionar tal como lo haría en el mundo profesional, fomentar la creatividad, ahorra tiempo y dinero, propicia la enseñanza individualizada, y facilita la autoevaluación (Salas y Ardanza, 1995; Mason y Rennie, 2006; Ruiz, 2008).

Según Castroviejo (2016), las características que aportan los simuladores al aprendizaje:

- Aprendizaje por descubrimiento. Se propone al alumno una serie de hipótesis sobre las que tiene que investigar, buscando unos efectos producidos por un fenómeno. Dentro de este tipo de aprendizaje es muy frecuente el sistema de prueba-error.

- Enseñanza individualizada. El alumno puede controlar su propio proceso de aprendizaje estableciendo los tiempos y las tareas a desarrollar al ritmo que le resulte más conveniente. Además, se puede repetir el proceso cuantas veces se desee hasta asimilar los conceptos necesarios.

- Desarrollo incremental de las habilidades. El alumno desarrollará sus competencias a partir del uso reiterado de la herramienta, implementando distintos modos y contextos de reproducir el fenómeno para obtener la destreza pretendida.

- Basado en casos reales. Ya sea a través de la realización de un proyecto o la resolución de problemas, esta herramienta tiene una fuerte fundamentación en los fenómenos reales.

- Autoevaluación. El sistema puede llevar implementado un sistema de autoevaluación. 
- Pedagógicamente neutros. Se trata de una herramienta que el docente puede emplear según le convenga, independientemente del método de enseñanza.

- Acompañamiento durante el proceso. El simulador en sí necesita de un apoyo externo para que tenga eficacia docente. El profesor juega un papel de guía, aportando información, de tal manera que sea suficiente para impulsar el proceso investigador y no sea exhaustiva para que los alumnos no lo sigan a modo de receta. Además, el docente debe cerciorarse de que se realiza el proceso de aprendizaje, aportando la información necesaria a través de otros canales.

Los simuladores de circuitos electrónicos son muy útiles para explicar el comportamiento de éstos de una forma sencilla a los alumnos de nivel superior en las asignaturas de Electricidad y Electrónica, el uso de la simulación por computadora es una herramienta imprescindible hoy en día a la hora de explicar los conceptos de electrónica en el aula, al ser la forma más sencilla y rápida de comprobar el funcionamiento de un circuito. Además, no necesita ningún tipo de material adicional para el montaje del mismo o medida de los resultados. Por supuesto, no se plantea el uso de los simuladores como alternativa al montaje real de los circuitos, sino como una herramienta complementaria que ayuda a comprender mejor el funcionamiento de los mismos.

El uso de la simulación por computadora es una herramienta imprescindible hoy en día a la hora de explicar los conceptos de electrónica en el aula, al ser la forma más sencilla y rápida de comprobar el funcionamiento de un circuito.

Tal y como lo menciona Montijano Moreno (2009), en la enseñanza de la electrónica, el uso de simuladores de circuitos es de mucha ayuda. Podemos señalar las siguientes ventajas:

- Permite ilustrar lecciones de teoría visualizando el comportamiento de componentes electrónicos, fuentes de alimentación e instrumentos de medida de una forma rápida y fácil de entender. 
- Facilita la visualización de señales difíciles de medir experimentalmente (por ejemplo, corrientes variables en el tiempo o varias tensiones y corrientes simultáneamente).

- Si el alumno conoce un simulador, puede comprobar por sí mismo si la solución que ha encontrado para un ejercicio propuesto es correcta comparándola con la del simulador.

- Permite que el alumno verifique sus propios diseños, testeando si funcionan antes de montarlos manualmente.

- Facilita el diseño de nuevos circuitos por parte de los alumnos.

- Ayuda a encontrar errores en los circuitos y diseños.

- Antes de montar un circuito en el laboratorio, la simulación del mismo facilita su análisis y comprensión.

Hasta ahora, las ventajas enumeradas están referidas únicamente al hecho de que los simuladores de circuitos favorecen de una u otra forma el proceso de enseñanza-aprendizaje en el campo de la electrónica. Sin embargo, el conocimiento acerca del uso de un simulador de circuitos es importante por sí mismo ya que le puede servir al alumno durante su vida profesional si ésta se desarrolla en empresas dedicadas al diseño y fabricación de productos electrónicos. Por ello, resulta conveniente que durante su formación el alumno del nivel superior en las asignaturas de Electricidad y Electrónica conozca no sólo los simuladores más sencillos (orientados a la docencia), sino que también se familiarice con los entornos de simulación más completos y profesionales.

A continuación, se presentan algunos de los principales software de simulación electrónica que actualmente son utilizados tanto en las instituciones de educación superior: 
- Multisim: es un software estándar en industria para diseño de circuitos y simulación SPICE para electrónica de potencia, analógica y digital en la educación y la investigación. integra simulación SPICE estándar en la industria con un entorno esquemático interactivo para visualizar y analizar al instante el comportamiento de los circuitos electrónicos. Su interfaz intuitiva ayuda a los profesores a reforzar la teoría de circuitos y a mejorar la retención de la teoría en todo el plan de estudios de ingeniería. Al añadir simulación potente de circuitos y análisis al flujo de diseño, Multisim $^{\mathrm{TM}}$ ayuda a los investigadores y diseñadores a reducir las iteraciones de prototipos de tarjeta de circuito impreso (PCB) y a ahorrar los costos del desarrollo (National Instruments, 2018).

- Livewire: Es un paquete de software sofisticado para diseñar y simular circuitos electrónicos.

Interruptores, transistores, diodos, circuitos integrados y cientos de otros componentes pueden conectarse entre sí para investigar el comportamiento de un circuito. No hay límites a lo que se puede diseñar y no hay conexiones sueltas ni componentes defectuosos de los que preocuparse. Sin embargo, si se exceden las especificaciones máximas para cualquier componente, jexplotarán en la pantalla! (New Wave Concepts, 2018).

- Proteus: Proteus Design Suite es una solución de software completa para simulación de circuitos y diseño de PCB. Comprende varios módulos para la captura esquemática, el firmware IDE y el diseño de PCB que aparecen como pestañas dentro de una aplicación única e integrada. Esto proporciona un flujo de trabajo ágil para el ingeniero de diseño y ayuda a que los productos lleguen al mercado más rápido. Todos los productos de simulación Proteus utilizan el módulo de captura esquemática como circuito electrónico y nuestro motor SPICE de modo mixto personalizado para ejecutar la simulación. Proteus VSM permite que el microcontrolador también se simule en el esquema, mientras que Proteus IoT Builder permite el diseño y la prueba de la interfaz de usuario remota para el circuito (LabCenter, 2018). 
- Micro-Cap: Micro-Cap 12 es un editor de esquemas integrado y un simulador mixto analógico / digital que proporciona un bosquejo interactivo y un entorno de simulación para los ingenieros electrónicos. Desde su lanzamiento original en 1982, Micro-Cap se ha ampliado y mejorado constantemente. Micro-Cap 12, la duodécima generación, combina una interfaz moderna e intuitiva con algoritmos numéricos robustos para producir niveles incomparables de potencia de simulación y facilidad de uso. Nada más se acerca (Spectrum Software, 2018).

\section{CONCLUSIONES}

La integración del pensamiento computacional en los currículos académicos es algo real en otros países que están cambiando la brecha digital y proporcionando los recursos necesarios para que las instituciones educativas cuenten con las herramientas tecnológicas y también capacitando a sus profesores en este nuevo reto de mejoras las habilidades del siglo XXI. Este nuevo enfoque educativo complementa los distintos modelos educativos ya que hace uso del pensamiento lógico al hacer uso de las matemáticas para interactuar con el mundo real, permitiendo que con el uso de dispositivos informáticos se puedan crear simulaciones en mundos virtuales en diferentes áreas del conocimiento.

Un ejemplo claro de lo anterior es La Hora del Código el cual es un movimiento global, que llega a decenas de millones de estudiantes en más de 180 países. Cualquier persona, en cualquier lugar del mundo puede organizar una Hora del Código. Los tutoriales, de una hora de duración, están disponibles en más de 30 idiomas. No se necesita experiencia. La Hora del Código es una introducción de una hora de duración a las Ciencias de la Computación, diseñada para mostrar que todo el mundo puede aprender a programar y así comprender los fundamentos básicos de la disciplina.

Otra aplicación del pensamiento computacional son los softwares de simulación electrónica que cobran gran relevancia dentro de los procesos de enseñanza y aprendizaje, ya que con ellos es posible analizar, comprender y experimentar sin la necesidad de contar con un laboratorio de pruebas que, en la mayoría 
de los casos en sumamente costoso. Para la rama de la electrónica en la última década se han desarrollado diferentes softwares de simulación que se han estado integrando como parte de la didáctica en las escuelas de educación superior en las Asignaturas de corte tecnológico, dentro de las cuales podemos encontrar: Análisis de Circuitos; Electrónica Analógica, Electrónica Digital, Microcontroladores y Dispositivos Lógicos Programables.

Además, se infiere que las principales ventajas de la simulación son las siguientes:

- Más facilidad para comprender los contenidos de la electrónica.

- Mayor facilidad de acceso que un taller o laboratorio.

- Versatilidad y facilidad de modificación de las premisas de partida.

- Focalización sobre el fenómeno de estudio en vez de en fórmulas matemáticas.

- Visualización y medición en tiempo real de las magnitudes físicas.

- Adaptación al ritmo del alumno.

- Fomento de la creatividad.

- Ahorro de tiempo y dinero.

Por otro lado, se reconoce que existen desventajas de la simulación:

- No son un método en sí. Necesitan el acompañamiento del docente.

- Los simulares tienen limitaciones para un aprendizaje completo de la materia.

- Necesitan ser desarrollados para adaptarlos al medio educativo. 


\section{REFERENCIAS BIBLIOGRÁFICAS}

Adell, J., Llopis, M.A., Esteve, F., y Valdeolivas, G. (2018). El debate sobre el pensamiento computacional en educación. Revista Iberoamericana de Educación a Distancia, 171-186. doi: https:// doi.org/10.5944/ried.22.1.22303

Arenas, M. (2017). NOBBOT Tecnologia para las personas. Recuperado el 2 de Diciembre de 2018, de https://www.nobbot.com/pantallas/miniaturizacion-tecnologica/

Asociacion de Profesores de Informatica de la Comunidad Valenciana. (2018). Conselleria d'Educació, Investigació, Cultura i Esport. Recuperado el 27 de Mayo de 2019, de www.ceice.gva. es/documents/162783553/162784550/bases_hora_codigo.pdf/c79f46b1-ede0-4982-859448b8ecb2344d

Barba, M. (2018). Blogthinkbig.com. Recuperado el 13 de Mayo de 2019, de https://blogthinkbig.com/ pensamiento-educacion

Gárdenas Peralta, M. G. (2019). Gobierno de México. Recuperado el 2019, de https://www.gob.mx/ cms/uploads/attachment/file/417818/Marco_de_Referencia_-_Pensamiento_Computacional. pdf

Contreras Gelves, G. A., García Torres, R., y Ramírez Montoya, M. S. (2010). Uso de simuladores como recurso digital para la transferencia de conocimiento. Apertura. Revista de innovación educativa, 2(1). Recuperado de http://www.udgvirtual.udg.mx/apertura/index.php/apertura/article/ view $/ 22$

Cortés Rojas, G., y García Santiago, S. G. (2012). Métodos de investigación I: Investigación documental. Veracruz, Mexico: Universidad Popular Autonoma de Veracruz. 
GSTA \& ISTE . (1 de Diciembre de 2011). EDUTEKA. Recuperado el 13 de Mayo de 2019, de https:// eduteka.icesi.edu.co/modulos/9/284/2062/1?url=9/284/2062/1

Ferro Soto, G., y Martínez Senra, A. I. (2009). Ventajas del uso de las TICs en el proceso de enseñanza-aprendizaje desde la óptica de los docentes universitarios españoles. Revista Electrónica de Tecnología Educativa, 29. doi: https://doi.org/10.21556/edutec.2009.29.451

Jurado Rosas, Y. (2005). Tecnicas de Investigacion Documental. Mexico: Thomson.

LabCenter. (2018). Proteus. Recuperado el 11 de Diciembre de 2018, de https:/ /www.labcenter.com/

López, S., Veit, E. A., y Araujo, I. S. (2016). Una revisión de literatura sobre el uso de modelación y simulación computacional para la enseñanza de la física en la educación básica y media. Revista Brasileira de Ensino de Física, 38(2), e2401. doi: http://dx.doi.org/10.1590/1806-9126RBEF-2015-0031

Moreno León, J. (2014). Programamos. Recuperado el 13 de Mayo de 2019, de https://programamos. es/que-es-el-pensamiento-computacional/

National Instruments. (2018). National Instruments. Recuperado el 11 de Diciembre de 2018, de http:// www.ni.com/es-mx/shop/electronic-test-instrumentation/application-software-for-electronictest-and-instrumentation-category/what-is-multisim.html

New Wave Concepts. (2018). New Wave Concepts. Recuperado el 11 de Diciembre de 2018, de https:// www.new-wave-concepts.com/ed/livewire.html

Pareja Aparicio, M. (2013). Software libre y simulación de circuitos electrónicos. Revista Digital de ACTA, 13. 
Rosario, J. (2006). TIC: Su uso como Herramienta para el Fortalecimiento y el Desarrollo de la Educación Virtual. Recuperado de http:/ / www.cibersociedad.net/archivo/articulo.php?art=221

Spectrum Software. (2018). Spectrum Software. Recuperado el 11 de Diciembre de 2018, de http:// www.spectrum-soft.com/demo.shtm

Téllez Ramírez, M. (2019). Pensamiento computacional: una competencia del siglo XXI. Educación Superior - Revista Científica de Publicación del Centro Psicopedagógico y de Investiganción en Educación Superior, 6(1), 23-32. Recuperado el 2019, de http://www.scielo.org:bo/scielo.php?script=sci_ arttext\&pid $=$ S2518-82832019000100007\&lng=es\&tlng=es

Valverde Berrocoso, J., Fernández Sánchez, M. R., y Garrido Arroyo, M. D. G. (2015). El pensamiento computacional y las nuevas ecologías del aprendizaje. RED-Revista de Educación a Distancia, 46. Recuperado de https://revistas.um.es/red/article/view/240311

Vilanova, G. E. (2018). Tecnología Educativa para el Desarrollo del Pensamiento Computacional. SISTEMAS, CIBERNÉTICA E INFORMÁTICA, 15(3), 25-32. Recuperado de http://www.iiisci. org/journal/CV\$/risci/pdfs/CA074QW17.pdf

Zapata-Ros, M. (2018). Pensamiento computacional desenchufado. Blog RED de Hypotheses. El aprendizaje en la Sociedad del Conocimiento. Recuperado el 2019, de https://red.hypotheses.org/1508

Zapata-Ros, M. (2015). Pensamiento computacional: Una nueva alfabetización digital . RED-Revista de Educación a Distancia, 46.

Zapotecatl López, J. L. (2018). Introducción al pensamiento computacional: conceptos básicos para todos. Mexico: Academia Mexicana de Computación, A. G. 